\title{
Siderophore production by Aeromonas salmonicida
}

\author{
Ian D. Hirst, ${ }^{*}$ Trevor S. Hastings and Anthony E. Ellis \\ DAFS Marine Laboratory, Victoria Road, Aberdeen AB9 8DB, UK
}

(Received 26 Octoher 1990; revised 20 December 1990; accepted 10 January 1991)

\begin{abstract}
Growth under conditions of iron-restriction and the production of siderophores was examined in 21 typical and 14 atypical strains of Aeromonas salmonicida. With the exception of one atypical strain, all strains grew and multiplied in the presence of the high-affinity iron chelators ethylenediamine di(o-hydroxyphenylacetic acid), $\alpha, \alpha^{\prime}-$ dipyridyl or transferrin. Chrome azurol $S$ agar was used to screen bacterial strains growing under these conditions for the production of siderophores. Siderophore production was detected only in the typical strains. Siderophores were also detected in the iron-restricted culture supernatants of typical strains, where they were associated with an iron-binding activity. The siderophore was extracted from iron-restricted culture supernatant of one strain by adsorption onto an XAD-7 resin; it behaved as a 2,3-diphenol-catechol in several colorimetric assays. The results indicate that although both typical and atypical strains of $A$. salmonicida grow and multiply under conditions of iron-restriction, they use different iron-uptake mechanisms, siderophore-mediated and siderophoreindependent, respectively. In cross-feeding assays, growth of typical strains was stimulated only by homologous iron-restricted supernatant, suggesting strain differences in the siderophore produced. However, one strain produced a culture supernatant with growth-stimulating activity for other typical and also atypical strains.
\end{abstract}

\section{Introduction}

Aeromonas salmonicida is the causative agent of furunculosis, a commercially important disease of cultivated salmonid fish. Typical strains of $\boldsymbol{A}$. salmonicida (subspecies salmonicida) produce a systemic disease generally restricted to salmonids which results in a chronic or acute haemorrhagic septicaemia, often with extensive liquefaction of host tissues (McCarthy \& Roberts, 1980). Atypical strains of $A$. salmonicida (subspecies achromogenes) produce a more superficial ulcerative form of furunculosis in the salmonids, ulcer disease in goldfish and erythrodermatitis in commercially-reared carp (Paterson, 1983).

To produce systemic disease, $A$. salmonicida must overcome non-specific defence mechanisms such as lysozyme, anti-proteases and complement (Ellis, 1981). Possession of a surface protein array, the $49 \mathrm{kDa}$ A-layer, by virulent strains of $A$. salmonicida is thought to enable the bacterium to overcome many of these defence mechanisms (Kay et al., 1981; Munn et al., 1982). An additional defence mechanism involves the binding of free iron to proteins such as transferrin to create iron-

\footnotetext{
Abbreviations: CAS, chrome azurol S; dd, deionized distilled; 2,3DHBA, 2,3-dihydroxybenzoic acid; EDDA, ethylenediamine di(ohydroxyphenylacetic acid); TLC, thin-layer chromatography.
}

restricted conditions within the host. Because iron is essential for bacterial growth and multiplication, $A$. salmonicida must express high-affinity iron-uptake mechanisms which can compete with the host iron-binding proteins to obtain sufficient iron for growth (Griffiths et al., 1988; Martinez et al., 1990). The best-understood iron-uptake mechanism possessed by pathogenic bacteria involves the production of siderophores. These are soluble, low-molecular-mass, iron-chelating molecules, which normally belong to two distinct chemical classes: phenolate-catechol siderophores, for example enterobactin, a cyclic triester of 2,3-dihydroxy- $N$-benzoyl serine; and hydroxamate siderophores, for example aerobactin, a conjugate of 6- $N$-acetyl- $N$-hydroxyamino-2-aminohexanoic acid and citric acid (Griffiths, 1987).

In an earlier study of $A$. salmonicida growing under conditions of iron-restriction, Chart \& Trust (1983) found that typical strains were able to sequester iron from a number of high-affinity iron chelators by two mechanisms. One mechanism appeared to involve the production of a siderophore(s). The siderophore activity was detected by its ability to sequester ${ }^{59} \mathrm{Fe}^{3+}$ from lactoferrin across a dialysis membrane with a $14 \mathrm{kDa}$ exclusion limit. Atypical strains did not appear to produce a siderophore. The siderophore was undetectable by the chemical assays for catechol and hydroxamate siderophores. The other mechanism - seen in only one 
typical strain - involved a direct contact between the cell surface and an iron-binding protein without the production of a siderophore, although the strain was able to utilize the siderophore-containing culture supernatant produced by a typical strain.

The present study describes the growth and production of siderophores by $A$. salmonicida under conditions of iron-restriction and reports attempts to extract the siderophore from culture supernatant and its partial chemical and biochemical characterization.

\section{Methods}

Bacterial strains. Twenty-one typical and 14 atypical strains of $A$. salmonicida were used in this study (Table 1). Three typical strains and one atypical strain were non-autoagglutinating and were presumed to lack the $49 \mathrm{kDa}$ A-layer. All other $A$. salmonicida strains were autoagglutinating. Other bacteria used in this study are listed in Table 2.

Culture media and conditions. Bacteria were grown either in tryptone soya broth (TSB, Oxoid) or on tryptone soya agar (TSA, Oxoid) with the exception of Vibrio anguillarum, which was grown in TSB and on TSA supplemented with $2 \%(\mathrm{w} / \mathrm{v})$ sodium chloride. All chemicals, unless otherwise stated, were obtained from Sigma. Ethylenediamine $\mathrm{di}\left(o\right.$-hydroxyphenylacetic acid) (EDDA $\left.; 100 \mu \mathrm{g} \mathrm{ml}^{-\mathrm{I}}\right)$, bovine transferrin $\left(5.0 \mathrm{mg} \mathrm{m}^{-1}\right)$ and $\alpha, \alpha^{\prime}$-dipyridyl $(100 \mu \mathrm{M})$ were added to induce conditions of iron-restriction. Contaminating iron was removed from EDDA by the method of Rogers (1973) and from transferrin by the method of Aisen et al. (1978). The iron content of the various media was determined by a ferrozine assay as described by Holzberg \& Artis (1983). The addition of EDDA, $\alpha, \alpha^{\prime}$-dipyridyl or transferrin reduced the free-iron content of the media to less than $0.025 \mu \mathrm{g} \mathrm{ml}^{-1}$ from an initial $0.385 \mu \mathrm{g} \mathrm{ml}^{-1}$. Bacteria were subcultured three times on the ironrestricted media before use, to reduce internal ferric iron stores. $A$. salmonicida, A. hydrophila, $A$. sobria and $V$. anguillarum strains were grown at $22^{\circ} \mathrm{C}$. E. coli and Ent. cloacae strains were grown at $37^{\circ} \mathrm{C}$. Growth of $A$. salmonicida in liquid media was monitored by measurement of $\mathrm{OD}_{540}$. All glassware was washed in $6 \mathrm{M}-\mathrm{HCl}$ and rinsed 10 times in deionized-distilled (dd) water obtained from a Milli $Q$ water purification system (Millipore) to remove contaminating iron.

Detection of the iron-binding activity of $A$. salmonicida culture supernatant. Iron-binding activity of $\boldsymbol{A}$. salmonicida culture supernatant was measured by the method of Norrod \& Williams (1978). Uninoculated culture medium, dd water or $48 \mathrm{~h}$ iron-restricted culture supernatant of four typical and two atypical strains was added to ironfree bovine transferrin solution $\left(2.5 \mathrm{mg} \mathrm{m}^{-1}\right.$ in $\left.0.1 \mathrm{M}-\mathrm{Tris} / \mathrm{HCl}\right)$ and incubated at $22^{\circ} \mathrm{C}$ for $1 \mathrm{~h}$. The binding of iron by transferrin in the presence or absence of a siderophore was determined by measuring $A_{470}$ after each addition of $20 \mu \mathrm{l}$ of $1.0 \mathrm{~mm}$-ferric nitrilotriacetate.

Siderophore assays. Bacteria were screened for the production of siderophores by the method of Schwyn \& Neilands (1987) using chrome azurol S (CAS) agar. Possible catechol siderophore production was assessed by the colorimetric assays of Arnow (1937) and Rioux et al. (1983), using 2,3-dihydroxybenzoic acid (2,3-DHBA) as standard. Possible hydroxamate siderophore production was assessed in lyophilized 10 -fold concentrated culture supernatant by the colorimetric assay of Csáky (1948) and in unconcentrated culture supernatant by the assays of Atkin et al. (1970) and Arnold \& Viswanatha (1983), using hydroxylamine monohydrochloride and desferrioxamine mesylate (Desferal; Ciba-Geigy) as standards. Crude siderophore preparations were analysed by paper and thin-layer chromatography (TLC). Paper
Table 1. Strains of Aeromonas salmonicida

\begin{tabular}{lll}
\hline Strain & Origin & Source
\end{tabular}

Typical (non-autoagglutinating)

MT004 Atlantic salmon Unilever Research Laboratory

MT532 (strain 2862)

$\begin{array}{lll}\text { MT539 Not known } & \text { C. Michel (strain 51/79S) }\end{array}$

Atypical

MT534 Carp D. Evenberg (strain V75/93-W)

Typical (autoagglutinating)

$\begin{array}{lll}\text { MT028 Brown trout } & \text { D. H. McCarthy (strain As-3) }\end{array}$

MT048 Atlantic salmon Marine Laboratory

MT423 Atlantic salmon S. Adams (strain 184/86)

MT476 Atlantic salmon Marine Laboratory

MT477 Atlantic salmon Marine Laboratory

MT478 Atlantic salmon Marine Laboratory

MT481 Atlantic salmon Marine Laboratory

MT483 Atlantic salmon Marine Laboratory

MT486 Atlantic salmon Marine Laboratory

MT487 Atlantic salmon Marine Laboratory

MT488 Atlantic salmon Marine Laboratory

MT489 Atlantic salmon Marine Laboratory

MT490 Atlantic salmon Marine Laboratory

MT491 Atlantic salmon Marine Laboratory

MT492 Atlantic salmon Marine Laboratory

MT537 Not known C. Michel (strain 36/75R)

MT538 Not known C. Michel (strain 72/78R)

MT616 Salmonid $\quad$ D. Evenberg (strain 152-69)

Atypical

MT194 Atlantic salmon Marine Laboratory

MT460 Sea trout Marine Laboratory

MT482 Atlantic salmon Marine Laboratory

$\begin{array}{lll}\text { MT523 Eel } & \text { T. Kitao (strain KF 8318) }\end{array}$

MT525 Atlantic salmon B. K. Gudmundsdottir (strain 265/87)

MT526 Atlantic salmon B. K. Gudmundsdottir (strain LON 82)

MT527 Atlantic salmon B. K. Gudmundsdottir (strain LON 76)

MT528 Atlantic salmon B. K. Gudmundsdottir (strain 510/SU)

MT529 Atlantic salmon B. K. Gudmundsdottir (strain 17/87)

MT530 Sea trout $\quad$ B. K. Gudmundsdottir (strain Fae II)

$\begin{array}{lll}\text { MT533 Atlantic salmon } & \text { B. K. Gudmundsdottir (strain Eldi 80) }\end{array}$

MT535 Goldfish $\quad$ D. Evenberg (strain 3.111)

MT536 Carp D. Evenberg (strain V76/135)

* Unilever Research Laboratories, Bedford, UK; C. Michel, Laboratoire d'Ichtyopathologie, Jouy-en-Josas, France; D. Evenberg, Institute of Molecular Biology and Department of Molecular Cell Biology, University of Utrecht, The Netherlands; D.H. McCarthy, Tavolek Inc., Redmond, Washington, USA; S. Adams, Institute of Aquaculture, University of Stirling, Stirling, UK ; T. Kitao, Department of Fisheries, Faculty of Agriculture, Miyazaki University, Japan; B. K. Gudmundsdottir, Institute for Experimental Pathology, Fish Disease Laboratory, University of Iceland, Reykjavik, Iceland.

chromatography was performed as described by Lemos et al. (1988), using butanol/acetic acid/water (120:30:30, by vol.) or $5 \%(\mathrm{w} / \mathrm{v})$ ammonium formate in $0.5 \%(\mathrm{v} / \mathrm{v})$ formic acid as solvent, and chromatograms were developed with the CAS assay solution of Schwyn \& Neilands (1987). TLC was carried out using methanol/water $(20: 15$, $\mathrm{v} / \mathrm{v})$ to detect anguibactin, the siderophore produced by $V$. anguillarum (Actis et al., 1986) and butanol saturated with $1.7 \%(w / v)$ ammonium acetate to detect amonabactin, one of the siderophores produced by $A$. hydrophila (Barghouti et al., 1989). Chromatographed material was visualized by its blue fluorescence under UV light and exposure to $2.7 \%$ $(\mathrm{w} / \mathrm{v})$ ferric chloride in $2 \mathrm{M}-\mathrm{HCl} / 3 \cdot 7 \%(\mathrm{w} / \mathrm{v})$ potassium ferricyanide spray. $R_{F}$ values were calculated using 2,3-DHBA as reference. 
Table 2. Strains of other bacteria used in this study

\begin{tabular}{|c|c|c|}
\hline Bacterium & Strain & Source* \\
\hline Aeromonas hydrophila $\dagger$ & B32 & L. A. Rodriguez \\
\hline Aeromonas hydrophila $\dagger$ & B5! & L. A. Rodriguez \\
\hline Aeromonas hydrophila & 1108 & NCIMB \\
\hline Aeromonas hydrophila ${ }^{\dagger}$ & 80-Al & L. A. Rodriguez \\
\hline Aeromonas sobriat & P-261 & L. A. Rodriguez \\
\hline Escherichia coli† & 9001 & NCTC \\
\hline Escherichia coli†§ & 50147 (K12 pColV-K 270) & NCTC \\
\hline Enterobacter cloacae $\dagger$ & 11580 & NCTC \\
\hline Vibrio anguillarum\| & $2286(775$ pJM1) & NCIMB \\
\hline
\end{tabular}

${ }^{*}$ L. A. Rodriguez, Microbiologia, Colegia Universitario de Orense, Orense, Spain; NCIMB, National Collection of Industrial and Marine Bacteria, Aberdeen, UK: NCTC, National Collection of Type Cultures, PHLS, London, UK.

$\dagger$ Enterobactin biosynthesis - positive Arnow assay in ethyl acetate extract of culture supernatant.

$\ddagger$ Amonabactin biosynthesis - positive Arnow assay in culture supernatant only

$\S$ Aerobactin biosynthesis - positive Csáky assay.

$\|$ Anguibactin biosynthesis - positive Arnow assay in culture supernatant only.

Siderophore extraction. Five different solvent extraction procedures were used to extract the siderophore of A. salmonicida MT004 from iron-restricted culture supernatant. The colorimetric assays of Arnow (1937) and Csáky (1948) were used to indicate the success of the extraction procedure. A $1 \mathrm{ml}$ sample of each extract was then evaporated under nitrogen and diluted five-fold in $\mathrm{dd}$ water for use in bioassays described later.

For ethyl acetate, diethyl ether and chloroform extractions, $5 \mathrm{ml}$ culture supernatant was adjusted to $\mathrm{pH} 1.5$ by addition of $1 \mathrm{M}-\mathrm{HCl}$ and extracted with $2.5 \mathrm{ml}$ solvent. The organic layer was collected, evaporated under nitrogen to dryness, and the residue dissolved in $1 \mathrm{ml}$ dd water.

For chloroform/phenol/diethyl ether extraction, $25 \mathrm{ml}$ culture supernatant was lyophilized, redissolved in $2.5 \mathrm{ml}$ dd water, adjusted to $\mathrm{pH} 2.0$ with $1 \mathrm{M}-\mathrm{HCl}$ and extracted with $20 \mathrm{ml}$ chloroform/phenol $(1: 1$, $\mathrm{v} / \mathrm{v})$. The organic layer was collected and diethyl ether $(15 \mathrm{ml})$ added, mixed and extracted with $10 \mathrm{ml}$ dd water. The aqueous extract was washed with ether, adjusted to $\mathrm{pH} 7.0$ with $1 \mathrm{M}-\mathrm{NaOH}$ and the aqueous extract evaporated under nitrogen to dryness. The residue was dissolved in $1 \mathrm{ml} \mathrm{dd}$ water.

For methanol extraction, $40 \mathrm{ml}$ methanol was added to the residue obtained from the lyophilization of $250 \mathrm{ml}$ of culture supernatant. The mixture was stirred at room temperature overnight and then centrifuged to remove undissolved material. The supernatant was evaporated under nitrogen and then suspended in $5 \mathrm{ml}$ dd water.

Partial purification of the A. salmonicida MT004 siderophore. Attempts to extract a siderophore from the iron-restricted culture supernatant of A. salmonicida MT004 were made by the method of Actis et al. (1986). Amberlite XAD-7 resin was washed with methanol, acetonitrile and diethyl ether to remove residual organic impurities prior to packing into a $2.5 \mathrm{~cm} \times 10 \mathrm{~cm}$ column in dd water. A 5 litre volume of culture supernatant adjusted to $\mathrm{pH} 7.0$ was applied to the XAD-7 column at a rate of 10 bed volumes per hour. The column was rinsed with $100 \mathrm{ml} \mathrm{dd}$ water followed by a step gradient of $25 \mathrm{ml}$ each of $33 \%(\mathrm{v} / \mathrm{v})$ and $66 \%$ $(\mathrm{v} / \mathrm{v})$ methanol/water mixtures. Adsorbed material was eluted with $75 \mathrm{ml}$ pure methanol. Peak column fractions, determined by the colorimetric assay of Arnow (1937), were evaporated under nitrogen and diluted five-fold in respect of an original volume of $\mathrm{lml}$ in dd water for use in the bioassays described later. A small amount of the adsorbed material was dissolved in a minimum volume of methanol and tested for purity on Silica G TLC plates. The plates were developed with the following solvent systems: butanol/acetic acid/water $(120: 30: 30$, by vol.): chloroform/methanol/water $(35: 12: 2$, by vol.); methanol/ammonium hydroxide $(100: 1 \cdot 5, \mathrm{v} / \mathrm{v})$; and ethyl acetate/methanol/acetic acid $(80: 1: 1$, by vol.). Chromatograms were visualized as described above.

Siderophore bioassay. The bioassay (Miles \& Khimji, 1975) measured the size of a growth halo around a $3 \mathrm{~mm}$ diameter well containing $30 \mu \mathrm{l}$ sample on plates containing TSA plus $200 \mu \mathrm{g}$ EDDA ml-1 which had been seeded with approximately $10^{5}$ colony-forming units (c.f.u.) of one of the six $\boldsymbol{A}$. salmonicida strains studied in this experiment. The diameter of the halo was measured after growth for $48 \mathrm{~h}$ at $22^{\circ} \mathrm{C}$, and the value corrected by subtraction of the $3 \mathrm{~mm}$ diameter of the sample well. The samples included culture supernatant of $A$. salmonicida, $A$. hydrophila, $A$. sobria and $V$. anguillarum, solvent extracts of $A$. salmonicida MT004 culture supernatant and peak column fractions of

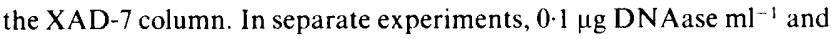
$0.1 \mu \mathrm{g} \mathrm{RNAase} \mathrm{ml}^{-1}$ were added to the culture supernatants in order to minimize the possibility of plasmid DNA transfer being implicated in bacterial growth. The siderophore activity of several catechols were examined in a similar manner, as were the culture supernatants of known siderophore-producing bacterial strains (see Table 2).

\section{Results}

\section{Growth of $A$. salmonicida under conditions of iron- restriction}

With the exception of one non-autoagglutinating atypical strain, all the strains of $A$. salmonicida tested grew and multiplied under conditions of iron-restriction. The growth rates of a typical and an atypical strain monitored in liquid medium containing the various iron chelators are represented in Fig. 1. In all cases of iron-restriction, growth rates were similar to those observed in normal liquid medium and liquid medium supplemented with $100 \mu \mathrm{M}$-ferric chloride. Minimal growth of A. salmonicida was observed on TSA containing $200 \mu \mathrm{g}$ EDDA $\mathrm{ml}^{-1}$ unless an additional source of iron was provided. This feature formed the basis of the siderophore bioassays in which culture supernatants of several strains of $A$. salmonicida were evaluated for their ability to reverse iron-restricted growth.

\section{Iron-binding activity of $A$. salmonicida culture supernatant}

The iron-binding activity of culture supernatant was examined in a typical and an atypical strain of $A$. salmonicida (Fig. 2). The presence of iron-binding activity was detected in the iron-restricted culture supernatant of typical strains by the ability of the culture supernatant to inhibit binding of ferric nitrilotriacetate to iron-free bovine transferrin. This inhibition occurred 


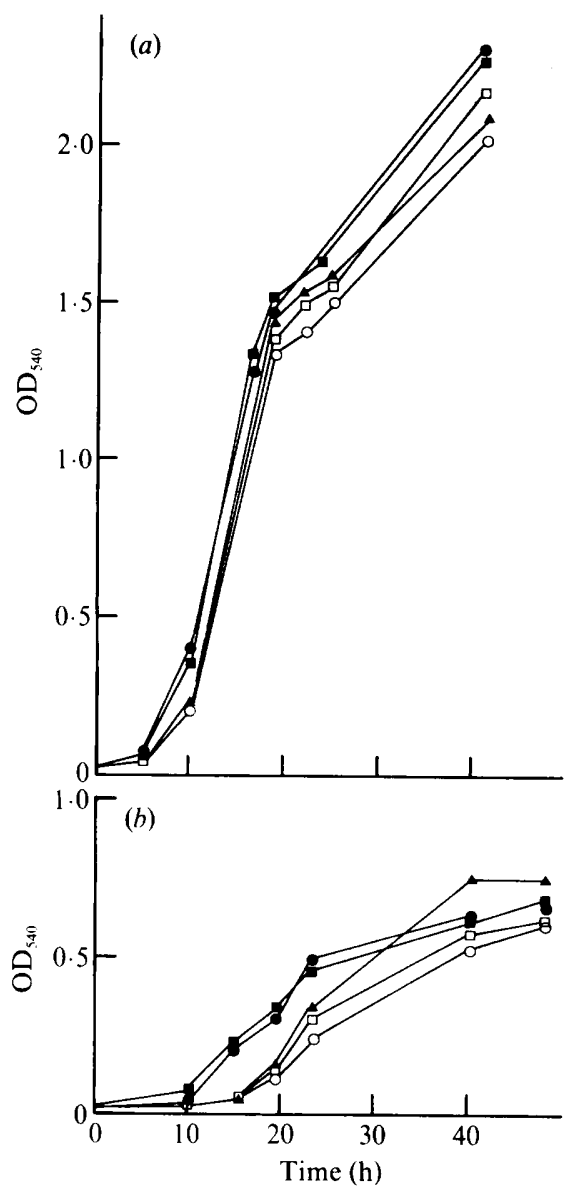

Fig. 1. Growth characteristics of $A$. salmonicida in TSB (O), TSB + $\mathrm{FeCl}_{3}(100 \mu \mathrm{M} ; \mathbf{\square}), \mathrm{TSB}+\operatorname{EDDA}\left(100 \mu \mathrm{g} \mathrm{ml}^{-1} ; \mathbf{A}\right)$, TSB $+\alpha, \alpha^{\prime}-$ dipyridyl $(100 \mu \mathrm{M} ; \mathrm{O})$ and TSB $+\operatorname{transferrin}\left(5 \mathrm{mg} \mathrm{ml}^{-1} ; \square\right)$. (a) Typical strain, MT004; (b) atypical strain, MT194.

with iron-restricted culture supernatants but not ironsufficient ones. Culture supernatants of the atypical strains failed to inhibit this reaction and showed no ironbinding activity.

\section{Production of siderophores}

All strains of $A$. salmonicida were screened for siderophore production using CAS agar (Schwyn \& Neilands, 1987); siderophore production was confined to the typical strains of $A$. salmonicida.

Culture supernatants of the 21 typical and 14 atypical strains were assayed colorimetrically for the presence of phenolate-catechol and hydroxamate siderophores. Small quantities of a catechol siderophore were detected by the method of Arnow (1937) in iron-restricted culture supernatants of 20 of the 21 typical strains. The same catechol siderophore was detected in all 21 strains by the
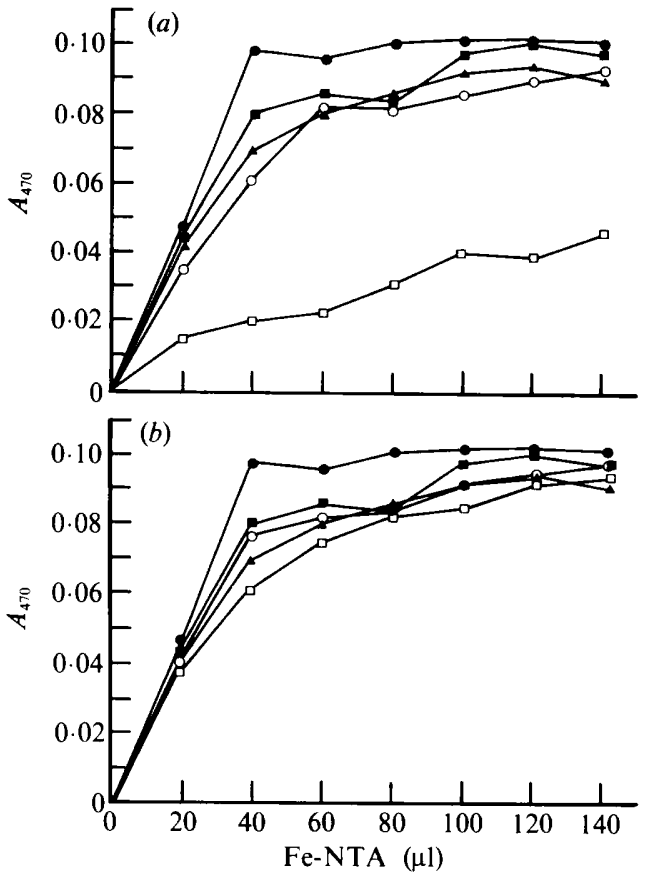

Fig. 2. Effects of culture supernatant from $A$. salmonicida on the binding of iron by transferrin. Culture supernatant and iron-free transferrin were incubated for $1 \mathrm{~h}$ at $22^{\circ} \mathrm{C}$. The binding of iron by transferrin was measured at $470 \mathrm{~nm}$ after each addition of $20 \mu \mathrm{l}$ ferric nitrilotriacetate (Fe-NTA). Symbols: $\boldsymbol{\bullet}$, water; $\boldsymbol{\square}$, TSB $\boldsymbol{\Delta}$, TSB + EDDA ; $\bigcirc$, culture supernatant from $A$. salmonicida; $\square$, iron-restricted culture supernatant from $A$. salmonicida. (a) Typical strain MT004: (b) atypical strain MT194.

method of Rioux et al. (1983). Small amounts of catechol were also detected in non-iron-restricted culture supernatants of typical strains by the assay of Rioux et al. (1983) but not by the assay of Arnow. These catechols were only detected in the culture supernatant of the typical strains but they were inactive in the siderophore bioassays. Culture supernatants of atypical strains were negative in the siderophore assays. The colorimetric assays of Csáky (1948), Arnold \& Viswanatha (1983) and Atkin et al. (1970) for the detection of hydroxamate siderophores were negative for both iron-restricted and non-ironrestricted culture supernatant of both typical and atypical strains of $A$. salmonicida.

Crude preparations of siderophore in iron-restricted culture supernatant were analysed by paper chromatography and TLC. Both analyses failed to detect any siderophore activity in $A$. salmonicida, although 2,3DHBA was detected in the culture supernatants of the typical strains. TLC was, however, successful in demonstrating the siderophores enterobactin, anguibactin and amonabactin in the culture supernatant of the appropriate siderophore-producing bacterial strains. 
Extraction and partial purification of the A. salmonicida MT004 siderophore

An attempt to extract the catechol siderophore produced by the typical strain MT004 was made by extraction of acidified culture supernatants with ethyl acetate, diethyl ether, chloroform, methanol and chloroform/phenol/ diethyl ether. The extraction procedures were unsuccessful as determined by colorimetric methods and bioassay. The catechol siderophore could be detected in acidified culture supernatant after these extractions by the assays of Arnow (1937) and Rioux et al. (1983), indicating that the siderophore remained in the aqueous phase.

A catechol siderophore was successfully extracted by adsorption onto XAD-7 resin. A comparison of the Arnow assay and siderophore bioassay before and after passage of the supernatant through the XAD-7 resin indicated that the resin adsorbed the siderophore. Elution of the XAD-7 resin with pure methanol resulted in a well-defined peak of siderophore bioassay activity which coincided with the Arnow assay peak activity (Fig. 3). However, much of the Arnow-positive material eluted earlier with a lower concentration of methanol, indicating elution of catechols with no siderophore activity. TLC of the pooled peak fractions (fractions 1435) using butanol/acetic acid/water revealed the presence of six components possessing phenolic groups $\left(R_{F}\right.$ $0.16,0.33,0.39,0.46,0.54$ and 0.73 ). Treatment of the pooled peak fractions and 2,3-DHBA with ferric chloride/potassium ferricyanide produced an intense blue colour characteristic of phenols containing vicinal di-hydric groups (Dawson et al., 1989). In an attempt to extract the siderophore from developed TLC plates, the six components were scraped from the plate, eluted with methanol and each component tested in the siderophore bioassay. All the components were negative in the bioassay, suggesting that the siderophore had undergone decomposition on the silica gel support medium.

\section{Cross-feeding}

The ability of the siderophore(s) present in the ironrestricted culture supernatant of typical or atypical strains of $A$. salmonicida to cross-feed other strains was examined using a siderophore bioassay (Table 3 ). Noniron-restricted culture supernatant or uninoculated medium failed to support the growth of any strain. The typical strains utilized factors from their own culture supernatant to stimulate growth under conditions of iron-restriction but only one strain (typical MT423) was able to stimulate growth of other typical and also the atypical strains. Culture supernatant of the atypical strains failed to stimulate their own growth or the growth of the typical strains under condition of iron-restriction.

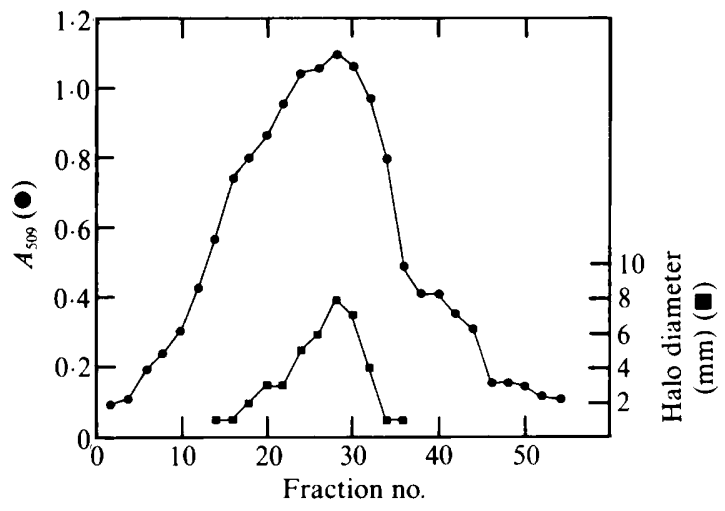

Fig. 3. Partial purification of the $A$. salmonicida MT004 siderophore on an XAD-7 column. Fractions of $2 \mathrm{ml}$ were collected, screened using the assay of Arnow (1937) (O) and diluted five-fold for the bioassay ( $\square$ ). Fractions $1-10$ were collected in $33 \%(\mathrm{v} / \mathrm{v})$ methanol, fractions $11-21$ in $66 \%(\mathrm{v} / \mathrm{v})$ methanol and fractions $22-55$ in $100 \%(\mathrm{v} / \mathrm{v})$ methanol.

Table 3. Summary of the ability of iron-restricted culture supernatants of four typical strains of $A$. salmonicida to cross-feed two atypical strains of $A$. salmonicida using a siderophore bioassay

The bioassay involved measuring the zone of growth around a $3 \mathrm{~mm}$ diameter well containing $30 \mu \mathrm{l}$ sample on a plate containing TSA plus $200 \mu \mathrm{g}$ EDDA ml ${ }^{-1}$ seeded with $10^{5}$ c.f.u. of the $\operatorname{six} A$. salmonicida strains. The results are the means of triplicate values.

\begin{tabular}{lcccccc}
\hline \hline & \multicolumn{7}{c}{$\begin{array}{c}\text { Diameter (mm) of zone of growth stimulated by } \\
\text { iron-restricted culture supernatant of strain }\end{array}$} \\
\cline { 2 - 7 } $\begin{array}{c}\text { Strain } \\
\text { incorporated } \\
\text { in agar* }\end{array}$ & $\begin{array}{c}\text { MT004 } \\
\text { (T) }\end{array}$ & $\begin{array}{c}\text { MT616 } \\
\text { (T) }\end{array}$ & $\begin{array}{c}\text { MT194 } \\
\text { (A) }\end{array}$ & $\begin{array}{c}\text { MT525 } \\
\text { (A) }\end{array}$ & $\begin{array}{c}\text { MT423 } \\
\text { (T) }\end{array}$ & $\begin{array}{c}\text { MT486 } \\
\text { (T) }\end{array}$ \\
\hline MT004(T) & 6 & 0 & 0 & 0 & 17 & 0 \\
MT616(T) & 0 & 18 & 0 & 0 & 15 & 0 \\
MT194(A) & 0 & 0 & 0 & 0 & 15 & 0 \\
MT525(A) & 0 & 0 & 0 & 0 & 14 & 0 \\
MT423(T) & 0 & 0 & 0 & 0 & 12 & 0 \\
MT486(T) & 0 & 0 & 0 & 0 & 10 & 17 \\
\hline \hline
\end{tabular}

*(T), typical; (A), atypical.

The addition of DNAase and RNAase to the culture supernatant had no effect on the growth of the various strains under iron-restriction. Culture supernatants of $E$. coli NCTC 9001 and NCTC 50147, Ent. cloacae NCTC 1 1580, A. hydrophila B32 and 80-A1, and A. sobria P-261, all containing enterobactin, promoted growth of typical and atypical strains of $A$. salmonicida to a small extent, although aerobactin, present in the culture supernatant of $E$. coli NCTC 50147 in addition to enterobactin, failed to promote further growth than enterobactin alone (Tables 4 and 5). Culture supernatants of $A$. hydrophila 1108 containing amonabactin and $V$. anguillarum 2286 (775 pJM1) containing anguibactin were unable to promote the growth of typical or atypical strains of $A$. salmonicida (Table 5). 
Table 4. Summary of the ability of iron-restricted culture supernatants of E. coli and Ent. cloacae to stimulate growth of six A. salmonicida strains grown under conditions of ironrestriction

Results are the means of triplicate values.

\begin{tabular}{cccc}
\hline \hline & \multicolumn{2}{c}{$\begin{array}{c}\text { Diameter (mm) of zone of growth stimulated by } \\
\text { iron-restricted culture supernatant of }\end{array}$} \\
\cline { 2 - 4 } $\begin{array}{c}\text { Strain } \\
\text { incorporated } \\
\text { in agar* }\end{array}$ & $\begin{array}{c}\text { E. coli } \\
\text { NCTC 9001 }\end{array}$ & $\begin{array}{c}\text { E. coli } \\
\text { NCTC 50147 }\end{array}$ & $\begin{array}{c}\text { Ent. cloacae } \\
\text { NCTC 11580 }\end{array}$ \\
\hline MT004(T) & 3.7 & 2.3 & 5.0 \\
MT616(T) & 4.0 & 3.7 & 5.7 \\
MT194(A) & 5.0 & $2 \cdot 3$ & 4.0 \\
MT525(A) & 4.0 & 3.0 & 2.7 \\
MT423(T) & 5.0 & 4.0 & 4.7 \\
MT486(T) & 4.7 & 2.7 & 4.0 \\
\hline \hline
\end{tabular}

*(T), typical; (A), atypical.

Table 5. Summary of the ability of iron-restricted culture supernatants of $A$. hydrophila, A. sobria and $V$. anguillarum to stimulate growth of six $A$. salmonicida strains grown under conditions of iron-restriction

Results are the means of triplicate values.

\begin{tabular}{llr}
\hline \hline & $\begin{array}{c}\text { Diameter (mm) of zone of } \\
\text { growth stimulated by iron- } \\
\text { restricted culture super- } \\
\text { natant of } \text { A. hydrophila }\end{array}$ \\
\cline { 2 - 3 } $\begin{array}{c}\text { Strain of } \\
\text { A. salmonicida* }\end{array}$ & B32 & $80-\mathrm{A} 1$ \\
\hline MT004(T) & 1.3 & 1.7 \\
MT616(T) & 1.7 & 1.7 \\
MT194(A) & 1.7 & 2.0 \\
MT525(A) & 1.3 & 1.3 \\
MT423(T) & 2.0 & 1.7 \\
MT486(T) & 1.7 & 1.7 \\
\hline \hline
\end{tabular}

*(T), typical; (A), atypical.

$\dagger$ Iron-restricted culture supernatants of $A$. hydrophila $1108, A$. sobria $\mathrm{P}-261$ and $V$. anguillarum $2286(775 \mathrm{pJM} 1$ ) did not stimulate the growth of any of the six $A$. salmonicida strains.

A number of catechols and iron-chelating compounds were tested in the same bioassays for their ability to promote or inhibit growth of $A$. salmonicida under conditions of iron-restriction. The compounds included 3,4-dihydroxyphenylalanine and 2,4,5-trihydroxyphenylalanine (6-hydroxy-dopa) - thought to be intermediates of pigment biosynthesis (Shieh \& Maclean, 1974; Donlon et al., 1983), catechol (1,2-dihydroxy benzene) and 2,3-DHBA. None of these compounds were able to promote the growth of $A$. salmonicida under conditions of iron-restriction.

\section{Discussion}

All of the strains examined in this study, with the exception of one non-autoagglutinating atypical strain, were able to grow and multiply in the presence of various high-affinity iron chelators where the available iron concentration was less than $0.025 \mu \mathrm{g} \mathrm{ml}^{-1}$. This suggests that both typical and atypical strains of $A$. salmonicida possess an effective high-affinity iron-uptake mechanism(s). The most common high-affinity iron-uptake mechanism possessed by pathogenic bacteria involves production of siderophores (Griffiths et al., 1988). Chart $\&$ Trust (1983) found the mechanisms by which typical and atypical strains of $A$. salmonicida acquired iron under conditions of iron-restriction to be varied (see Introduction). In the present study, using a universal chemical assay (CAS agar) for siderophore production, to screen both typical and atypical strains of $A$. salmonicida grown under conditions of iron-restriction, we found that only the typical strains produced a siderophore. An ironbinding activity able to chelate ferric iron was also detected in the iron-restricted culture supernatant of the typical strains. No siderophore production or ironbinding activity was detected in the atypical strains, leading us to believe that atypical strains of $A$. salmonicida acquire iron by a siderophore-independent mechanism.

The siderophore-like activity described by Chart \& Trust (1983) could not be detected by the assays used to detect catechol and hydroxamate siderophores. They used the assay of Arnow (1937) to detect catechol siderophores in ethyl acetate extracts of acidified culture supernatant. Although this procedure has been used to detect enterobactin (the siderophore produced by $E$. coli and other members of the Enterobacteriaceae) (O'Brien et al., 1970; O'Brien \& Gibson, 1970), the extraction depends upon the solubility of this siderophore, and most other catechols, in organic solvents at acidic $\mathrm{pH}$, due to the release of the chelated iron (Bezkorovainy, 1980). However, in the present study, the siderophore produced by $A$. salmonicida could not be extracted from culture supernatant into any of the organic solvents examined. Recently a phenolate siderophore, composed of 2,3DHBA, lysine, glycine and either tryptophan or phenylalanine, has been described in $A$. hydrophila (Barghouti al., 1989) which also could not be extracted into organic solvents (Liles et al., 1985). Similarly, anguibactin, the phenolate-containing siderophore from Vibrio anguillarum composed of $w$ - $N$-hydroxyhistamine, cysteine and 2,3-DHBA (Jalal et al., 1989), could not be isolated from culture supernatant by solvent extraction. With these two siderophores in mind, we examined the ironrestricted culture supernatant of $A$. salmonicida directly for the presence of a catechol siderophore and were able 
to detect a catechol siderophore using the assays of Arnow (1937) and Rioux et al. (1983). The siderophore was partially purified by adsorption onto an XAD-7 resin and found to behave as a 2,3-diphenolate-catechol. Its catechol nature was demonstrated from a positive reaction in the assay of Arnow (1937) and in the ferric catechol-phenanthroline assay of Rioux et al. (1983). The presence of a phenolic group was demonstrated by its blue fluorescence under UV light and its reaction with ferric chloride/potassium ferricyanide spray, which also suggests the catechol possesses vicinal di-hydric groups typical of diphenols (Dawson et al., 1989). A 2,3diphenol structure for the siderophore is probable since aromatic vic-diols with 4- or 5-substituents fail to react in the Arnow assay (Barnum, 1977). Negative reactions with the Csáky assay and ferric perchlorate assay of Atkin et al. (1970) indicated that the siderophore contained no hydroxamate functional groups. Although similar in chemical nature to anguibactin and amonabactin in being a 2,3-diphenol-catechol and insoluble in organic solvents, the $\boldsymbol{A}$. salmonicida siderophore had different chromatographic properties when analysed by TLC. Differences in the chemical nature of the siderophores were also observed in the siderophore bioassays, in which $A$. salmonicida was unable to utilize either anguibactin or amonabactin culture supernatants.

Small amounts of non-siderophore catechols present in non-iron-restricted culture supernatant of the typical strains were detected by the assay of Rioux et al. (1983). Their detection was probably due to the assay being seven times more sensitive than the Arnow assay which failed to detect their presence. Since both assays are selective for aromatic vic-diols, a wide variety of catechols may be detected such as catechol, 2,3-DHBA and 3,4-dihydroxyphenylacetic acid (Barnum, 1977). A number of the possible intermediates of pigment biosynthesis, characteristic of the typical strains, such as 3,4-dihydroxyphenylalanine and 2,4,5-trihydroxyphenylalanine (6-hydroxy-dopa) (Shieh \& Maclean, 1974; Donlon et al., 1983), are aromatic vic-diols. It is possible that the non-siderophore catechols detected in non-ironrestricted culture supernatant at lower concentrations than seen in iron-restricted culture supernatant are the intermediates of pigment biosynthesis. The presence of 2,3-DHBA was detected by TLC in the iron-restricted culture supernatant of typical strains of $A$. salmonicida. None was detected in the iron-restricted culture supernatant of the atypical strains. The presence of 2,3-DHBA is characteristic of bacteria which produce phenolatecatechol siderophores (Actis et al., 1986). The function of 2,3-DHBA is unclear since it fails to exhibit any siderophore activity for $A$. salmonicida in the siderophore bioassay.

The siderophore present in the iron-restricted culture supernatant of the typical strains of $A$. salmonicida stimulated growth of homologous strains. An interesting feature of the iron-restricted culture supernatant produced by typical strain MT423 was its ability to stimulate growth of heterologous strains, including the atypical strains. This may be due to an unidentified growthpromoting factor induced by the iron-restricted conditions in addition to a strain-specific siderophore, or to a slight difference in the chemical nature of the siderophore compared with that of $A$. salmonicida MT004. The ability of the MT423 iron-restricted culture supernatant to cross-feed heterologous strains suggests a similarity to the cross-feeding activity described by Chart \& Trust (1983), in which a typical strain possessing a siderophore activity cross-fed a non-siderophore-producing typical strain. However, in the present study, the MT423 ironrestricted culture supernatant also cross-fed the atypical strains. This suggests that both typical and atypical strains possess a receptor capable of utilizing siderophores present in iron-restricted culture supernatant, possibly the iron-regulated outer-membrane proteins described by Chart \& Trust (1983) and Aoki \& Holland (1985).

The $49 \mathrm{kDa}$ surface array protein (A-layer) of $A$. salmonicida has also been implicated as being a component of an iron-uptake mechanism, by Kay et al. (1985). They found the A-layer to possess a unique binding site for the porphyrins haemin and protoporphyrin IX, and speculated that the binding of porphyrins to the A-layer may represent an initial stage in iron uptake. Since it appears from this study that atypical strains of $A$. salmonicida acquire iron by a siderophore-independent process, the mechanism proposed by Kay et al. (1985) may play an important role in the acquisition of iron by A. salmonicida. This may especially be the case with the atypical strains, since their normally slow growth on normal laboratory media can be improved when sources of porphyrin such as haematoporphyrin and haemoglobin are added (Ishiguro et al., 1986).

This work was supported by a grant from the Crown Estate Commission. We thank Luis Rodriguez for provision of the $A$. hydrophila and $A$. sobria strains.

\section{References}

Actis, L. A., Fish, W. Crosa, J. H., Kellerman, K., Ellentberger S. R., Hauser, F. M. \& SANDERs-Loehr, J. (1986). Characterisation of anguibactin, a novel siderophore from Vibrio anguillarum (pJM1). Journal of Bacteriology 167, 57-65.

Aisen, P., Liebman, A. \& Zweier, J. (1978). Stoichiometric and site characteristics of the binding of iron to human transferrin. Journal of Biological Chemistry 253, 1930-1937.

AOKI, T. \& HOLLAND, B. I. (1985). The outer membrane proteins of the fish pathogens Aeromonas hydrophila, Aeromonas salmonicida and Edwardsiella tarda. FEMS Microbiology Letters 27, 299-305. 
ARNOLD, L. D. \& Viswanatha, T. (1983). The use of bis(mercaptoaceto- $S, O$ )hydroxoiron(III) complex for the determination of hydroxamates. Journal of Biochemical and Biophysical Methods 8, 307-320.

ARNOW, L. E. (1937). Colorimetric determination of the components of 3,4-dihydroxyphenylalanine-tyrosine mixtures. Journal of Biological Chemistry 118, 531-537.

Atkin, C. L., Neilands, J. B. \& Phaff, H. J. (1970). Rhodotorulic acid from species of Leucosporidium, Rhodosporidium, Rhodotorula, Sporidiobolus and Sporobolomyces, and a new alanine-containing ferrichrome from Cryptococcus melibiosum. Journal of Bacteriology 103, 722-733.

Barghouti, S., Young, R., Olson, M. O. J., Arceneaux, J. E. L., Clem, L. W. \& Byers, B. R. (1989). Amonabactin, a novel tryptophan- or phenylalanine-containing phenolate siderophore in Aeromonas hydrophila. Journal of Bacteriology 171, 1811-1816.

BARNUM, D. W. (1977). Spectrophotometric determination of catechol, epinephrine, dopa, dopamine and other aromatic vic-diols. Analytica Chimica Acta 89, 157-166.

Bezkorovainy, A. (1980). Biochemistry of Non-heme Iron. New York: Plenum.

Chart, H. \& Trust, T. J. (1983). Acquisition of iron by Aeromonas salmonicida. Journal of Bacteriology 156, 758-764.

CsÁky, T. Z. (1948). On the estimation of bound hydroxylamine in biological materials. Acta Chemica Scandinavica 2, 450-454.

Dawson, R. M. C., Elliot, D. C., Elliot, W. H. \& Jones, K. M. (1989). Phenols and related compounds. In Data for Biochemical Research, 3rd edn, pp. 482-485. Edited by R. M. C. Dawson, D. C. Elliot, W. H. Elliot \& K. M. Jones. Oxford: Clarendon Press.

Donlon, J., McGettigan, S., O’Brien, P. \& Ó'Carra, P. (1983). Reappraisal of the nature of the pigment produced by Aeromonas salmonicida. FEMS Microbiology Letters 19, 285-290.

ELlIS, A. E. (1981). Non-specific defence mechanisms in fish and their role in disease processes. Developments in Biological Standardisation 49, 337-352.

GriffiThs, E. (1987). The iron-uptake systems of pathogenic bacteria. In Iron and Infection - Molecular, Physiological and Clinical Aspects, pp. 69-137. Edited by J. J. Bullen \& E. Griffiths. Chichester: John Wiley.

Griffiths, E., Chart, H. \& Stevenson, P. (1988). High-affinity iron uptake systems and bacterial virulence. In Virulence Mechanisms of Bacterial Pathogens, pp. 121-137. Edited by J. A. Roth. Washington, DC: American Society for Microbiology.

HolzberG, M. \& ARTIS, W. M. (1983). Hydroxamate siderophore production by opportunistic and systemic fungal pathogens. Infection and Immunity 40, 1134-1139.

Ishiguro, E. E., Ainsworth, T., Kay, W. W. \& Trust, T. J. (1986). Heme requirement for growth of fastidious atypical strains of Aeromonas salmonicida. Applied and Entironmental Microbiology 51, 668-670.

Jalal, M. A. F., Hossain, M. B., van der Helm, D., Sanders-Loehr, J., Actis, L. A. \& Crosa, J. H. (1989). Structure of anguibactin, a unique plasmid-related bacterial siderophore from the fish pathogen Vibrio anguillarum. Journal of the American Chemical Society 11, 292-296.
Kay, W. W., Buckley, J. T., Ishiguro, E. E., Phipps, B. M., Monette, J. P. L. \& Trust, T. J. (1981). Purification and disposition of a surface protein associated with virulence of Aeromonas salmonicida. Journal of Bacteriology 147, 1077-1084.

Kay, W. W., Phipps, B. M., Ishiguro, E. E. \& Trust, T. J. (1985). Porphyrin binding by the surface array virulence protein of Aeromonas salmonicida. Journal of Bacteriology 164, 1332-1336.

Lemos, M. L., Salinas, P., Toranzo, A. E., BarJa, J. L. \& Crosa, J. H. (1988). Chromosome-mediated iron uptake system in pathogenic strains of Vibrio anguillarum. Journal of Bacteriology 170, 1920-1925.

Liles, D., Byers, B. R., Byers, P. E., Arceneaux, J. E. L. \& Lobb, C. (1985). Production of a siderophore and utilisation of transferrin-iron by Aeromonas hydrophila. Abstracts of the Annual Meeting of the American Society of Microbiology K179, 201.

Martinez, J. L., Delgado-Iribarren, A. \& Baquero, F. (1990). Mechanisms of iron acquisition and bacterial virulence. FEMS Microbiology Reviews 75, 45-56.

MCCARThy, D. H. \& Roberts, R. J. (1980). Furunculosis of fish - the present state of our knowledge. In Advances in Aquatic Microbiology, pp. 293-341. Edited by M. R. Droop \& H. W. Jannasch. London: Academic Press.

Miles, A. A. \& KHIMJi, P. L. (1975). Enterobacterial chelators of iron: their occurrence, detection and relation to pathogenicity. Journal of Medical Microbiology 8, 477-490.

Munn, C. B., Ishiguro, E. E., Kay, W. W. \& Trust, T. J. (1982). Role of surface components in serum resistance of Aeromonas salmonicida. Infection and Immunity 36, 1069-1075.

NorRod, P. \& Williams, R. P. (1978). Effects of iron and culture filtrates on killing of Neisseria gonorrhoeae by normal human serum. Infection and Immunity 21, 918-924.

O'BRIEN, I. G. \& GiBSON, F. (1970). The structure of enterochelin and related 2,3-dihydroxy- $N$-benzoylserine conjugates from Escherichia coli. Biochimica et Biophysica Acta 215, 393-402.

O'Brien, I. G., Cox, G. B. \& Gibson, F. (1970). Biologically active compounds containing 2,3-dihydroxybenzoic acid and serine formed by Escherichia coli. Biochimica et Biophysica Acta 201, 453-460.

Paterson, W. D. (1983). Furunculosis and other associated diseases caused by Aeromonas salmonicida. In Antigens of Fish Pathogens, pp. 119-137. Edited by D. P. Anderson, M. Dorsan \& P. H. Dubourget. Collection Fondation Marcel Merieux.

Rioux, C., Jordan, D. C. \& Rattray, J. B. M. (1983). Colorimetric determination of catechol siderophores in microbial cultures. Analytical Biochemistry 133, 163-169.

ROGERS, H. J. (1973). Iron-binding catechols and virulence in Escherichia coli. Infection and Immunity 7, 445-456.

SCHWYN, B. \& NEILANDS, J. B. (1987). Universal chemical assay for the detection and determination of siderophores. Analytical Biochemistry 160, 47-56.

Shieh, H. S. \& Maclean, J. R. (1974). Melanin biosynthesis by a fish pathogen, Aeromonas salmonicida. International Journal of Biochemistry 5, 643-647. 